The aim of the study was evaluating social determinants and gender differences in WLC.

Methods The study has been carried out in three workplaces (Call centre, plastic and metal industry) in 807 workers between 01.08.2016 and 01.01.2017. Independent variables were age, gender, maritual status, educational status and occupational class. Dependent variable was WLC. Copenhagen Psychosocial Questionnaire-3 were used to evaluate work life conflict. We analysed the association between social determinants and work life conflict by using chi-square test and logistic regression.

Results Mean age of men and women were 32.1 $7.4,27.2$ \pm 6.6 , respectively. $43.0 \%$ were women. WLC was found higher in women $(55.9 \%)$ than men $(42.4 \%) \quad(p<0.001)$. White collar, younger and high educated workers had higher risk of having WLC than their reference groups $(p<0.05$ for all comparisons). Logistic regression analysis revealed that, being younger and married were independently associated with WLC $(\mathrm{p}<0.05)$ in women. Being younger was independently associated with WLC $(p<0.05)$ in men. There were no association between educational status or occupational class and WLC in both gender.

Conclusion In this study, an inverse relationship was found between age of the employees and WLC. Marital status is important factor in women but not men in terms of WLC.

\section{INFLUENCE OF FACTORS OF WORK-LIFE BALANCE ON WORK ENGAGEMENT AMONG JAPANESE WORKERS}

${ }^{1} \mathrm{~K}$ Hayashi ${ }^{*},{ }^{2} \mathrm{~T}$ Nagata. ${ }^{1}$ Ewel, Inc., Japan; ${ }^{2}$ Institute of Industrial Ecological Sciences, University of Occupational and Environmental Health, Japan

\subsection{6/oemed-2018-ICOHabstracts. 1756}

Introduction Studies on work engagement (WE) tend to be related to work factors. Few studies exist on non-work factors such as work-life balance(WLB), living condition in family, and leisure time. Currently, Japan has experienced changes in demographic factors due to the declining birthrate and ageing population and in psychological factors due to diversification of sense of values. Moreover, as the time outside of working hours increases due to national policies on working style and working time management reform, intervention for non-work factors, especially factors of WLB, will become important for improving WE in the future.

Methods Data on 10060 persons among Japanese workers, collected using an employee questionnaire administered at the Collaboration Health Study Group (11 companies; 29194 persons), were analysed after excluding missing data. First, the Spearman's correlation coefficient was calculated to examine the relationship between WE and seven items related to factors of WLB (number of acquired paid vacation days, number of acquired paid vacation days by purpose, leisure time [estimated from overtime hours], degree of satisfaction of time allocation, recovery experiences, buffer factors other than the workplace, and sleeping hours). Items with high correlation coefficients were subjected to a single regression analysis.

Results Of the factors of WLB, a weak correlation was noted for degree of satisfaction with time allocation $(r=0.366)$. Moreover, a single regression analysis revealed the items in the order of degree of satisfaction with time allocation $(\beta=0.149)$.

Conclusion Among the factors of WLB, higher satisfaction with time allocation and subjective sense of health corresponded to higher WE. In future, we plan to continue to examine how to use time and leisure content, and to use the findings to develop concrete measures for occupational health and human resources management.

\section{THE ASSOCIATION BETWEEN WORK-FAMILY SPILLOVER AND QUALITY OF SLEEP: A PROSPECTIVE STUDY OF JAPANESE WORKERS}

${ }^{1}$ Yui Hidaka*, ${ }^{2}$ Akihito Shimazu, ${ }^{3}$ Kotaro Imamura, ${ }^{4}$ Noboru Iwata, ${ }^{5}$ Kyoko Shimada, ${ }^{6}$ Masaya Takahashi, ${ }^{7}$ Masahito Tokita, ${ }^{8}$ Izumi Watai, ${ }^{3}$ Kazuhiro Watanabe, ${ }^{3}$ Norito Kawakami. 'Department of Psychiatric Nursing, Graduate School of Medicine, The University of Tokyo, Tokyo, Japan; ${ }^{2}$ Center for Human Social Science, Kitasato University College of Liberal Arts and Science, Sagamihara, Japan; ${ }^{3}$ Department of Mental Health, Graduate School of Medicine, The University of Tokyo, Tokyo, Japan; ${ }^{4}$ Department of Psychology, Hiroshima International University, Hiroshima, Japan; ${ }^{5}$ Department of Social Psychology, Faculty of Sociology, Toyo University, Tokyo, Japan; ${ }^{6}$ National Institute of Occupational Safety and Health, Kawasaki, Japan; 'Department of Psychiatric and Mental Health Nursing, Graduate School of Nursing, Chiba University, Chiba, Japan; ${ }^{8}$ Department of Nursing, Graduate School of Medicine, Nagoya University, Nagoya, Japan

\subsection{6/oemed-2018-ICOHabstracts. 1757}

Introduction Quality of Sleep has been linked to poor health. While work-family spillover is associated with health problems and impaired work performance of workers, previous findings were inconsistent on, the association between work-family spillover and sleep quality. No study was conducted in nonwestern countries. The objective of this study was to investigate the prospective association between work-family spillover and sleep quality among Japanese workers.

Methods The database used in the study was from a 2 year prospective cohort study. In the baseline survey (T1) in 2011, questionnaires were sent to 1356 workers living in two wards of Tokyo, and 753 (56\%) responded, and 489 completed all scales and items used the study. A follow-up survey was conducted in 2013, with $224(46 \%)$ respondents out of the 489 completers at T1; 176 completed all scales. The questionnaire (both T1 and T2) included self-reported instruments of workfamily spillover (the Survey Work-home Interaction-NijmeGen, SWING), sleep quality (Pittsburgh Sleep Quality Index, PSQI), and job stressors (Brief Job Stress Questionnaire, BJSQ), and demographic variables (age, sex, education, marital status, and work styles). Multiple linear regression analysis was employed of PSQI scores on SWING scale scores, adjusting for demographic variables and PSQI score at T1. (SPSS version 22).

Results Among the 176 completers, 36\% were men; the average age was 39 years old. Work to family negative spillover (beta $=0.20)$ significantly and positively correlated with sleep quality, after adjusting for the demographic variables $(p=0.020)$. No significant association was observed between the other type of spillover (i.e. work to family positive, family to work positive or negative) and sleep quality. ( $p>0.05)$.

Conclusion This prospective study confirmed the association between work-family negative spillover and sleep quality in a sample of Japanese workers. Work to family negative spillover could be considered a target condition to improve sleep quality of workers. 